\title{
Caracterización y evaluación de la templabilidad de una fundición gris empleada en la fabricación de camisas de cilindro para motores diésel
}

\author{
Edgar L. Castellanos-Leal ${ }^{\mathrm{a}}$, Ana E. Coya , Jaime A. Gonzáleza , Olga P. Vesga Rueda ${ }^{\mathrm{b}}$, \\ David A. Miranda ${ }^{\mathrm{a}, \bigotimes}$ \\ ${ }^{a}$ Universidad Industrial de Santander, Sede Guatiguará, km 2, El Refugio, Piedecuesta Santander, Colombia \\ ${ }^{\mathrm{b}}$ Industrias LAVCO, Km 4 Autopista Floridablanca-Piedecuesta, Santander, Colombia \\ Autor para la correspondencia: dalemir@uis.edu.co
}

Enviado: 27 Noviembre 2016; Aceptado: 23 Abril 2017; Publicado on-Line: 1 Septiembre 2017

\begin{abstract}
RESUMEN: El aumento de las propiedades mecánicas (dureza superficial) de las camisas de cilindro es uno de los objetivos de las empresas de fundición especializadas, en miras de aumentar la competitividad de sus productos en el mercado local y extranjero. El objetivo de este estudio se centró en la caracterización de la fundición gris utilizada en la producción de las camisas y en la determinación de los parámetros metalúrgicos en el diseño del tratamiento térmico de temple convencional. Lo anterior, se realizó mediante la evaluación de la templabilidad del material por el método de Grossman y el ensayo Jominy, la selección de la temperatura de austenización y la severidad del medio de enfriamiento para un adecuado endurecimiento del material. Los resultados revelaron que el excelente valor de dureza obtenido luego del tratamiento es atribuido a la adecuada templabilidad de la fundición y a la severidad de temple seleccionada.
\end{abstract}

PALABRAS CLAVE: Camisas de cilindro; Fundición gris; Motores diésel; Temple convencional; Tratamientos térmicos

Citar como/Citation: Castellanos-Leal, E.L.; Coy, A.C.; González, J.A.; Vesga Rueda, O.P.; Miranda, D.A. (2017) "Caracterización y evaluación de la templabilidad de una fundición gris empleada en la fabricación de camisas de cilindro para motores diésel”. Rev. Metal. 53(3):e101. http://dx.doi.org/10.3989/revmetalm.101

\begin{abstract}
Characterization and hardenability evaluation of gray cast iron used in the manufacture of diesel engine cylinder liners. The increment of the mechanical properties (surface hardness) of engine cylinder is one of the principal goals for foundry company, to increase the competitiveness of their products in the local and foreign market. This study focused on the characterization of the gray cast iron used in the production of engine cylinder liners and metallurgical parameters determination in the design of conventional quenching heat treatment. The characterization was performed by material hardenability evaluation using Grossmann method, and Jominy test; the austenitizing temperature and the severity of cooling medium to a proper hardening of material were selected. Results revealed that the excellent hardness value obtained is attributed to the suitable hardenability of the gray cast iron and adequate severity selection for hardening treatment.
\end{abstract}

KEYWORDS: Diesel engines; Engine cylinder liners; Gray cast iron; Heat Treatments; Quenching

ORCID ID: Edgar L. Castellanos-Leal (http://orcid.org/0000-0001-7876-4404); Ana E. Coy (http://orcid.org/00000001-8813-0042); Jaime A. González (http://orcid.org/0000-0002-8611-7933); Olga P. Vesga Rueda (http://orcid. org/0000-0001-5391-4445); David A. Miranda (http://orcid.org/0000-0003-3130-3314)

Copyright: (C) 2017 CSIC. Este es un artículo de acceso abierto distribuido bajo los términos de la licencia Creative Commons Attribution (CC BY) España 3.0. 


\section{INTRODUCCIÓN}

En la actualidad, la industria metalmecánica en Colombia tiene una alta dependencia de compañías extranjeras debido al leve desarrollo tecnológico y a la carencia en innovación dentro del sector. Esta situación hace que se compren materias primas importadas debido a la poca capacidad de diversificación en las líneas de producción nacional y a la desigualdad en el costo de los productos traídos del exterior (DPN, 2007). Por esta razón, ha surgido la necesidad de implementar nuevos procesos de innovación apoyados en la academia, la industria y el Estado, impulsando el conocimiento de las nuevas tecnologías utilizadas, equipos de fabricación modernos, aseguramiento de la calidad y manufactura de una amplia gama de productos (Ferreira, 2002).

En las industrias de fundición, se requiere ampliar el horizonte del mercado de camisas para motores diésel. Este tipo de cilindros que son destinados al desplazamiento de los pistones en el motor de un automóvil, deben ser fabricados con una aleación fuerte para poder soportar a lo largo de su vida útil un trabajo de alta temperatura con explosiones constantes de combustible (Telejko et al., 2009; Balachandran et al., 2011). El material utilizado consiste principalmente en una fundición gris, que se caracteriza por tener buenas propiedades mecánicas junto con una buena resistencia al desgaste (Yamazaki et al., 2006). Sin embargo, para evitar el rápido deterioro y continuo reemplazo de la camisa, es necesario mejorar sus propiedades mecánicas. En este sentido, el tratamiento térmico de temple se convierte en una opción atractiva si se piensa en una resistencia mecánica mejorada y en evitar la utilización de otro tipo de material con propiedades superiores pero con elevados costos (Michalski y Wós, 2011). Aunque el endurecimiento por temple de una fundición es similar a la de los aceros, existen ciertas diferencias que deben ser manejadas con precaución, principalmente las condiciones de calentamiento y severidad de temple en el ciclo térmico, que son afectadas por la variada composición y la microestructura de las fundiciones (Maroni, 1976; Lasheras, 1978; Brooks, 1979).

Por lo anterior, es necesario realizar un estudio adecuado del material, incorporando un tratamiento térmico de endurecimiento efectivo, que permita diseñar un proceso optimizado teniendo en cuenta características importantes de la fundición gris, como su templabilidad, temperatura de austenización y el medio de temple. Todo lo anterior representa una atractiva incorporación tecnológica que podría incrementar las ventas de la empresa en un $10 \%$, con la consecuente generación de empleo, mejorar la competitividad y aumentar la representación en los mercados nacionales e internacionales.

\section{MATERIALES Y MÉTODOS}

En este trabajo, se empleó una muestra de una fundición gris (Industrias LAVCO Ltda) denominada CL, obtenida a través de un horno cubilote. Este tipo de fundición se utiliza para la fabricación de camisas de cilindro para motores diésel.

\subsection{Caracterización del material}

El análisis de composición química de la muestra en condiciones de colada se realizó por la técnica de espectrometría de emisión óptica en un equipo marca Bruker, referencia Q4 Tasman con arco de chispa en atmósfera de argón (Tabla 1).

La muestra CL se sometió a una preparación metalográfica, teniendo en cuenta la norma ASTM E3-11 (2011), que indica inicialmente un desbaste gradual con papel abrasivo P600 de carburo de silicio, hasta granulometría de $25,8 \mu \mathrm{m}$, seguido de un pulido con pasta de diamante de 3 y $1 \mu \mathrm{m}$ hasta obtener una superficie especular. El tipo, tamaño y distribución de grafito se determinó empleando la norma ASTM A247-16a (2016). Posteriormente, con objeto de revelar su microestructura, las muestras fueron atacadas con una solución de nital al 2\% durante 20 segundos según la norma ASTM E407-07 (2015), y su caracterización microestructural se llevó a cabo empleando un microscopio óptico OLYMPUS GX71.

\subsection{Medición de la templabilidad (Ensayo Jominy)}

Este ensayo consiste en un temple en agua que se realiza sobre uno de los extremos de una probeta cilíndrica con geometría definida, la medición de la respuesta al endurecimiento (dureza) se da en función de la distancia al extremo templado (Angus, 1976). La probeta fue previamente mecanizada y presentó las siguientes dimensiones: $25 \mathrm{~mm}$ de diámetro y $100 \mathrm{~mm}$ de longitud con una pestaña de $3 \mathrm{~mm}$ de espesor en uno de los extremos para asegurarla en el dispositivo de enfriamiento, según la norma ASTM A255-10 (2014). Inicialmente, se fijó un chorro de agua con un caudal y temperatura constantes. Luego la muestra se colocó en una mufla a una temperatura de austenización específica de $870{ }^{\circ} \mathrm{C}$ y se mantuvo a esta temperatura durante $30 \mathrm{~min}$. Posteriormente, la probeta

TABla 1. Composición química de la fundición estudiada (CL) expresada en porcentaje en peso

\begin{tabular}{lcccccccccc}
\hline Elemento & $\mathbf{C}$ & $\mathbf{S i}$ & $\mathbf{M n}$ & $\mathbf{C u}$ & $\mathbf{C r}$ & $\mathbf{S}$ & $\mathbf{N i}$ & $\mathbf{P}$ & $\mathbf{M o}$ & $\mathbf{C}_{\text {eqv }}$ \\
\hline CL $(\%)$ & 3,87 & 2,72 & 0,39 & 0,281 & 0,2 & 0,15 & 0,097 & 0,074 & 0,025 & 4,8 \\
\hline
\end{tabular}


Jominy fue removida del horno y se llevó rápidamente al dispositivo de enfriamiento. Finalmente, se colocó un flujo de agua constante sobre uno de sus extremos durante 10 min hasta llevarla a temperatura ambiente.

Después del proceso de temple, se mecanizaron dos generatrices opuestas sobre la superficie de la probeta (desbastando aproximadamente $0,4 \mathrm{~mm}$ ), para determinar los valores de dureza sobre el área plana en ciertos intervalos de distancia de $1,5 \mathrm{~mm}$ a partir del extremo templado.

\subsection{Parámetros metalúrgicos del tratamiento}

El rango de la temperatura crítica de transformación en este tipo de fundiciones está influenciada principalmente por su contenido de silicio, más que por el contenido de carbono y de los otros elementos aleantes (ASM Handbook, 1991). La temperatura más baja (inicio de la transformación) del rango crítico para las fundiciones puede estimarse mediante la siguiente expresión, la cual puede extenderse $55^{\circ} \mathrm{C}$ o más del valor obtenido:

Temperatura crítica,

${ }^{\circ} \mathrm{C}=730+28(\% \mathrm{Si})-25(\% \mathrm{Mn})$

Por otro lado, la severidad del temple $(\mathrm{H})$ es un variable indispensable en el control del tratamiento. Usualmente, para las fundiciones grises se recomienda el enfriamiento en aceite en lugar del realizado en agua debido a la buena respuesta observada en la dureza y a la disminución del riesgo de agrietamiento (Gliner y Vybornov, 2014). Para determinar la severidad de temple en el tratamiento de la fundición CL, se utilizaron cilindros de $25 \mathrm{~mm}$ de diámetro con una longitud suficientemente larga para despreciar la influencia del enfriamiento sobre las bases. A continuación, se describe el procedimiento llevado a cabo:

- Se coloca el cilindro de $25 \mathrm{~mm}$ de diámetro dentro de una mufla a $870{ }^{\circ} \mathrm{C}$ durante 1 hora.

- Se efectúa el temple en aceite a $25^{\circ} \mathrm{C}$ con agitación acentuada.

- Luego del temple, se corta un disco con un espesor de $12 \mathrm{~mm}$ y se determinan los valores de dureza a lo largo de la sección transversal con objetivo de obtener la curva en forma de "U".

- Se elige un punto de la curva "U" cuya distancia al centro $\left(r_{i}\right)$ guarde la relación $r_{i} / R=0,5$.

- De la curva "U" se obtiene el valor de dureza en el punto medio del radio del redondo.

- Con este valor de dureza se determina la distancia Jominy del extremo templado a la cual se produce la misma dureza.

- Finalmente, con el valor de la distancia y el diámetro del redondo $(25 \mathrm{~mm})$ se verifica en la curva de Lamont, la medida de la severidad de temple, $\mathrm{H}$.

\section{RESULTADOS Y DISCUSIÓN}

\subsection{Composición química}

En la Tabla 1, se muestran los resultados de la composición química de la fundición CL. Se puede observar la presencia de pequeñas cantidades de elementos de aleación (Cr, Ni, Mo y Mn), que podrían influir positivamente sobre su templabilidad (Gliner y Vybornov, 2014).

Usualmente, las fundiciones comerciales se consideran aleaciones ternarias $\mathrm{Fe}-\mathrm{C}-\mathrm{Si}$; a consecuencia de esto, las temperaturas críticas difieren de las presentadas por los aceros al carbono. Sin embargo, es importante mencionar el efecto que puede tener la presencia de los elementos aleantes sobre el proceso de temple. Por ejemplo, un contenido de silicio entre 0,6 y $3,0 \%$ genera desplazamientos en las curvas de transformación de fases (Valencia, 2009). Las adiciones del 1 al 2\% de níquel o cobre son particularmente efectivas en el incremento de la capa endurecida para una geometría y velocidad de temple determinadas (Grossmann, 1942). Por otra parte, la presencia de cromo y molibdeno es muy frecuente en las fundiciones para mejorar la resistencia mecánica de las piezas en el caso de productos con secciones simples, tales como camisas de cilindro, anillos del pistón, entre otros, debido a la formación de carburos de cromo y molibdeno. Sin embargo, ambos elementos al ser potentes formadores de carburos, aumentan en gran medida el riesgo de agrietamiento en las secciones críticas de piezas con geometría más compleja durante el enfriamiento rápido (Piyapong, 2007).

El contenido de carbono, silicio y fósforo se consideró para estimar el porcentaje de carbono equivalente (CE) en la fundición CL, usando la siguiente expresión (Davis, 1996), Ec. (2):

$\mathrm{CE}=\% \mathrm{C}+1 / 8 \% \mathrm{P}+1 / 3 \% \mathrm{Si}$

El CE establece la relación entre el efecto de los elementos aleantes en una fundición y la cantidad de carbono que se requeriría para obtener un efecto similar en el tratamiento térmico (Yamazaki et al., 2006). El valor obtenido de la expresión (Ec. (2)) fue 4,8, lo que indica que el material se clasifica como una fundición hipereutéctica (ASM Handbook, 1992; Vadiraj et al., 2011).

\subsection{Análisis metalográfico}

Las microestructuras de la fundición gris CL en condiciones de colada se muestran en las Figs. 1 y 2. En la Fig. 1 se presenta una micrografía sin ataque donde es posible notar la presencia de grafito 
laminar que, según la clasificación dada en la norma ASTM A247-16a (2016), corresponde a láminas tipo $\mathrm{C}$, rosetas de grafito y grafito eutéctico tipo B (subenfriado).

La profundidad de penetración en el endurecimiento depende del tipo de matriz y de la cantidad, tamaño y distribución del grafito en la fundición. La capa superficial endurecida (estructura martensítica), toma lugar principalmente por la transformación de la región perlítica de la fundición gris. Las zonas ferríticas presentes antes del calentamiento, pueden también transformarse en austenita con una adecuada temperatura de austenización, para que

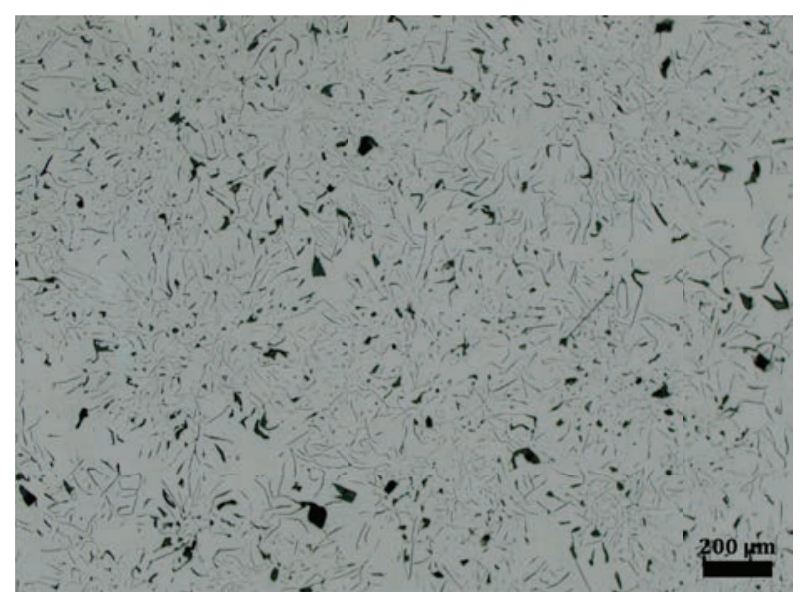

Figura 1. Micrografía óptica de la fundición gris CL, sin ataque químico a $100 \mathrm{X}$.

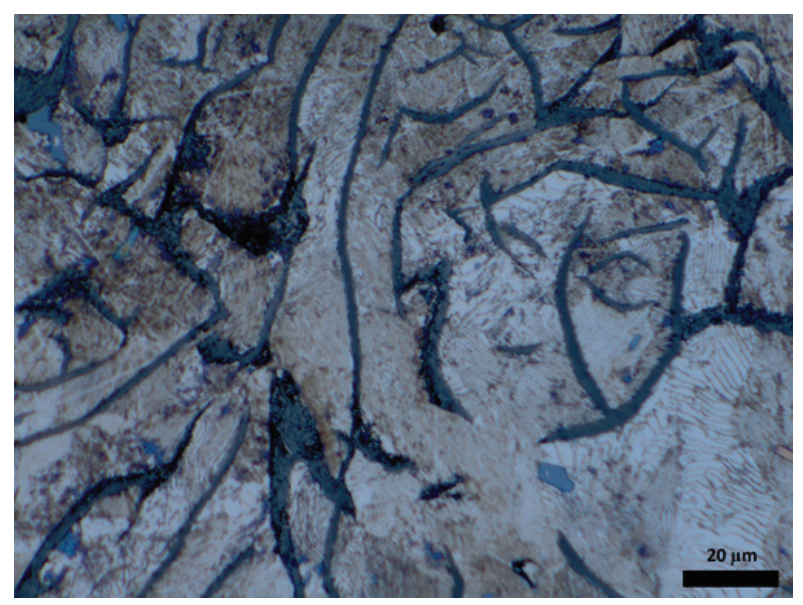

Figura 2. Micrografía óptica de la microestructura de CL, con ataque químico (solución $2 \%$ Nital) a $1000 \mathrm{X}$. esta última se transforme total o parcialmente en martensita (Ruiz et al., 1996; Rejowski et al., 2012). Por otra parte, la muestra que es objeto de estudio en este trabajo, posee una matriz perlítica con esteadita (eutéctico ternario de cementita, fosfuro de hierro y ferrita) y grafito laminar, con una distribución aproximada de 86,4 y $10 \%$ en peso, respectivamente, como se puede apreciar en la Fig. 2. Estas características morfológicas podrían proporcionar una rápida austenización, en comparación con una matriz predominantemente ferrítica, según lo mencionado por Malinochka (1963), ya que, al estar enriquecida con silicio, se satura más lentamente con carbono y, en consecuencia, el proceso de austenización se torna más complejo. El contenido de silicio presente en esta fundición permitió la obtención de una matriz totalmente perlítica. Sin embargo, si la cantidad de silicio fuese mayor, probablemente se generaría una matriz ferrítica-perlítica por el hecho de que el silicio es un fuerte grafitizador (Vadiraj et al., 2011), generando como consecuencia un porcentaje de carbono combinado menor de $0,5 \%$ (carbono disuelto en la austenita) y por ende una baja respuesta al temple.

\subsection{Templabilidad de la fundición gris}

Diámetro crítico ideal: La templabilidad es una propiedad que determina la profundidad del endurecimiento adquirido en una aleación al ser templada en un medio específico (Totten et. al., 1993). Dentro de las opciones para medir dicha característica, se encuentra el cálculo del diámetro crítico ideal, $D_{i}$, el cual se puede estimar cuantitativamente utilizando el método analítico de Grossmann (Grossmann, 1942), que relaciona los elementos de aleación con factores de multiplicación (ver Tabla 2). Elementos como Cr, $\mathrm{Mo}, \mathrm{Mn}, \mathrm{Si}, \mathrm{Ni}$ y $\mathrm{V}$ retrasan la transformación de fase de austenita a ferrita y perlita, debido a la necesidad de redistribución de estos elementos durante la transformación de fase difusional (Malinochka, 1963; Dimitry et at., 2016). Los elementos de aleación no sólo afectan la templabilidad, también se ha reportado su gran influencia en la selección de una apropiada temperatura de austenización y tiempo de sostenimiento del tratamiento térmico de temple. Por ejemplo, el silicio favorece la aparición de grafito laminar, disminuyendo la solubilidad del carbono y el contenido de carbono combinado (volumen de cementita) (ASM Handbook, 1992). Por otra parte, el manganeso promueve la formación de perlita, aumentando la solubilidad del carbono

TABLA 2. Factores de multiplicación asociados a la composición química de la fundición

\begin{tabular}{lccccccccc}
\hline Elemento & $\mathbf{C}$ & $\mathbf{S i}$ & $\mathbf{M n}$ & $\mathbf{C u}$ & $\mathbf{C r}$ & $\mathbf{S}$ & $\mathbf{N i}$ & $\mathbf{P}$ & $\mathbf{M o}$ \\
\hline$\%$ & 3,87 & 2,72 & 0,39 & 0,281 & 0,201 & 0,15 & 0,097 & 0,074 & 0,025 \\
Factor & 48 & 1,55 & 1,21 & - & 1,4 & - & 1,02 & 0,78 & 1,08 \\
\hline
\end{tabular}


y frenando la difusión de este en la austenita, lo que permite temperaturas de austenización más bajas (ASM Handbook, 1992). Además, los elementos como Mo y Cr son fuertes formadores de carburos, haciendo necesario el aumento de la temperatura de austenización y el tiempo de sostenimiento para disolver las fases de carburos presentes (Vadiraj et al., 2011).

El diámetro crítico ideal se estimó mediante la siguiente expresión (Ec. (3)):

$$
D_{i}=D_{A} * F_{S i} * F_{M n} * F_{C r} * F_{N i} * F_{P} * F_{M o}
$$

El cálculo de $D_{i}$ depende del valor de $D_{A}$ relacionado al porcentaje de carbono (Cunningham et al., 1999) en la fundición y de los factores multiplicadores de cada elemento de aleación. Las fundiciones no aleadas pueden tener valores de $D_{i}$ de $25 \mathrm{~mm}$, mientras que las aleadas pueden alcanzar los $100 \mathrm{~mm}$ o más (Cunningham et al., 1999). Para este caso, la fundición CL obtuvo un $D_{i}=108,29 \mathrm{~mm}$. Este valor representa una buena templabilidad, lo cual está relacionado con los resultados obtenidos en la caracterización microestructural de la fundición que también indican buenas condiciones estructurales para el proceso de austenización y por ende un efectivo proceso de temple.

Ensayo Jominy: Las mediciones de dureza con respecto a la distancia del extremo templado sobre la probeta Jominy corresponden a las sucesivas velocidades de enfriamiento logradas a lo largo de la probeta (temple diferencial) según la norma ASTM A255-10 (2014). Los resultados obtenidos se muestran en la Fig. 3.

Se puede observar que a medida que aumenta la distancia desde el extremo templado ocurre una disminución leve pero constante de la dureza que va desde $53 \mathrm{HRC}$ hasta $46 \mathrm{HRC}$ en los primeros $16 \mathrm{~mm}$ de la probeta. Sin embargo, desde ese punto, los valores de dureza caen drásticamente y siguen disminuyendo

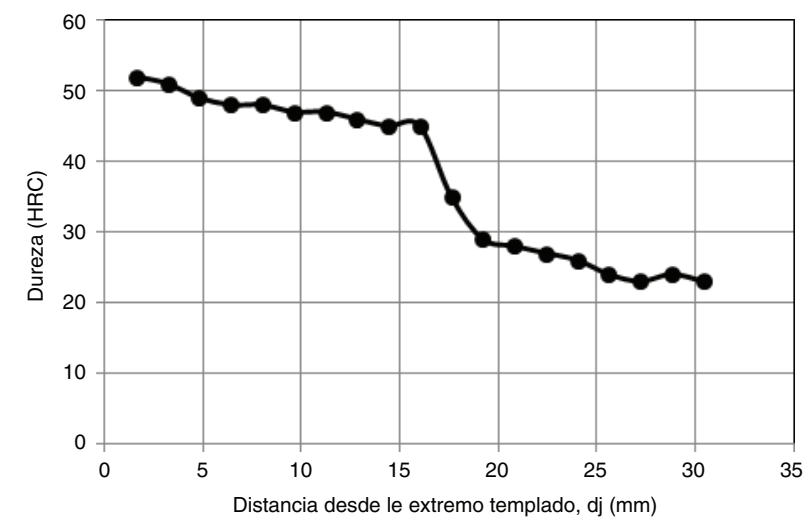

Figura 3. Curva de templabilidad Jominy para la fundición CL. hasta alcanzar cerca de $25 \mathrm{HRC}$. La ligera reducción inicial de dureza hasta $16 \mathrm{~mm}$ desde el extremo templado (de 53 a 46 HRC) corrobora los resultados previos, indicando una buena templabilidad de la fundición (Clarke y Van Tyne, 2005). En la siguiente sección se establecen las condiciones que se llevaron a cabo para obtener un ciclo térmico adecuado.

Otro método empleado en la determinación del $D_{i}$ (medida de la templabilidad), se basa en la utilización de la curva Jominy experimental. En el cual se selecciona la curva de Lamont $r_{i} / R=0,0$ que relaciona la distancia Jominy (dj) y un medio de enfriamiento ideal $\mathrm{H}=\infty$ (Lamont, 1943). Dicha correlación es adecuada, puesto que el $D_{i}$ como la curva de Jominy son función exclusivamente de la fundición (Composición química y tamaño de grano).

Para un 0,5\% de $\mathrm{C}$ disuelto en la austenita, se obtiene una dureza de la fundición correspondiente a un $50 \%$ de martensita, $\mathrm{HRC}=46$ (valor tomado de la curva dureza máxima vs $\% \mathrm{C}$ y $\%$ martensita (Bates y Totten, 1992)). Luego de la curva Jominy se obtiene una distancia $\mathrm{dj}=16 \mathrm{~mm}$ o $10 / 16$ de pulgada para esa misma dureza y finalmente, con la distancia Jominy obtenida y una velocidad de enfriamiento $\mathrm{H}=\infty$, se determina el diámetro critico ideal $D_{i}=90 \mathrm{~mm}$ o 3,6 in empleando la curva de correlación entre la velocidad de enfriamiento, distancia del extremo templado y diámetro de la barra descrita por Valencia (2009).

De lo anterior, se puede concluir que aunque el diámetro crítico ideal obtenido con la curva Jominy experimental ha resultado ser un $17 \%$ menor comparado con el valor calculado de forma teórica, esta variación se considera aceptable y normal, y debe tomarse en cuenta en el diseño del tratamiento térmico del material.

\subsection{Parámetros metalúrgicos para el tratamiento térmico de temple}

El rango de temperaturas típico para un proceso de endurecimiento por temple en fundiciones grises y dúctiles está entre 820 y $960^{\circ} \mathrm{C}$ (ASM Handbook, 1991). Utilizando la expresión (Ec. (1)) descrita en la sección experimental y sabiendo que, en la práctica se emplean temperaturas alrededor de $90{ }^{\circ} \mathrm{C}$ por encima de la calculada (Smolijan et al., 2006), se decidió realizar la austenización a una temperatura de $870^{\circ} \mathrm{C}$ y un tiempo de sostenimiento de $1 \mathrm{~h}$.

En relación con la determinación de la severidad de temple, H, la Fig. 4 muestra la curva en forma de "U". De la curva se obtiene un valor de dureza de $44 \mathrm{HRC}$, en el punto medio del radio del redondo a una distancia de $6,2 \mathrm{~mm}$ del centro del disco. Con el valor de dureza (44 HRC), se determinó la distancia Jominy al extremo templado correspondiente a 9,52 $\mathrm{mm}$, zona donde se produce el mismo valor de dureza. Finalmente, en la intersección entre el valor 


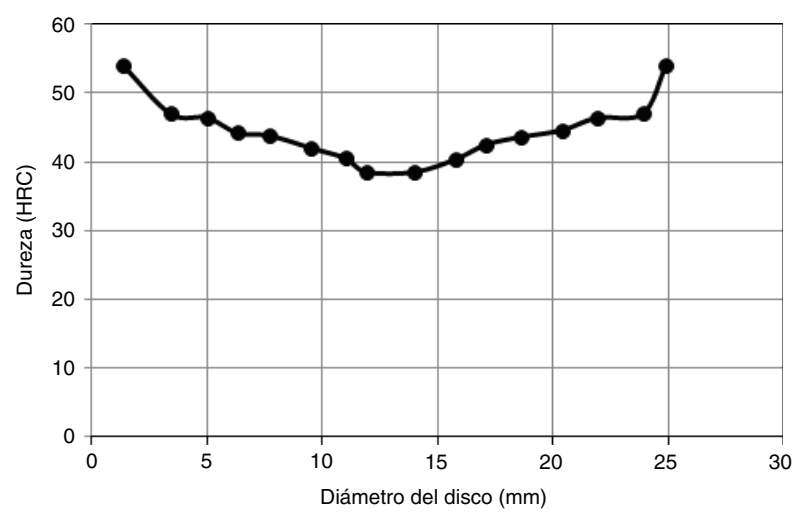

Figura 4. Curva "U" de un disco de $25 \mathrm{~mm}$ de diámetro templado en aceite con agitación acentuada.

de la distancia Jominy $(9,52 \mathrm{~mm})$, el diámetro del redondo $(25 \mathrm{~mm})$ y la curva de Lamont para una relación $r_{i} / R=0,5$, se encontró una severidad de temple $\mathrm{H}=0,4$.

Teniendo en cuenta los resultados obtenidos y que la dureza que se requiere en la superficie interior de este tipo de camisas es de $54 \mathrm{HRC}$, luego de ser templada, se determinó que el tratamiento térmico de temple que se debe llevar a cabo es el que se resume a continuación:

Austenización. El calentamiento de la fundición a $870{ }^{\circ} \mathrm{C}$ durante $1 \mathrm{~h}$, para alcanzar un tamaño de grano igual a $39,1 \mu \mathrm{m}$ (ASTM E7-15, 2015).

Enfriamiento. Temple por inmersión en aceite con agitación acentuada $(H=0,4)$.

Después del tratamiento térmico efectuado, se realizó el análisis microestructural de la fundición CL utilizada en la fabricación de camisas (ver Fig. 5). En las micrografías se pudo corroborar los resultados obtenidos en la templabilidad de la fundición gris, que se relacionan directamente con la buena dureza obtenida (54 HRC) luego del tratamiento. Se observó una estructura típica de una fundición gris hipereutéctica, compuesta principalmente por martensita revenida (estructura acicular fina) junto con una cantidad de aproximadamente $4 \%$ de austenita retenida (tonalidad blanca) y láminas de grafito no disuelto (Vadiraj et al., 2011). Lo anterior, demuestra que es posible efectuar un proceso de endurecimiento adecuado sobre el material, y finalmente, se puede pensar en la fabricación de camisas para motores con resistencia mecánica mejorada a través de un tratamiento térmico de temple.

\section{CONCLUSIONES}

- La presencia de pequeñas cantidades de elementos de aleación convenientemente seleccionados, tales como $\mathrm{Cr}, \mathrm{Ni}, \mathrm{Mo}$ y $\mathrm{Mn}$ en la fundición $\mathrm{CL}$, ejercen una influencia efectiva sobre la templabilidad y por ende en la capacidad de endurecimiento. Adicionalmente, la

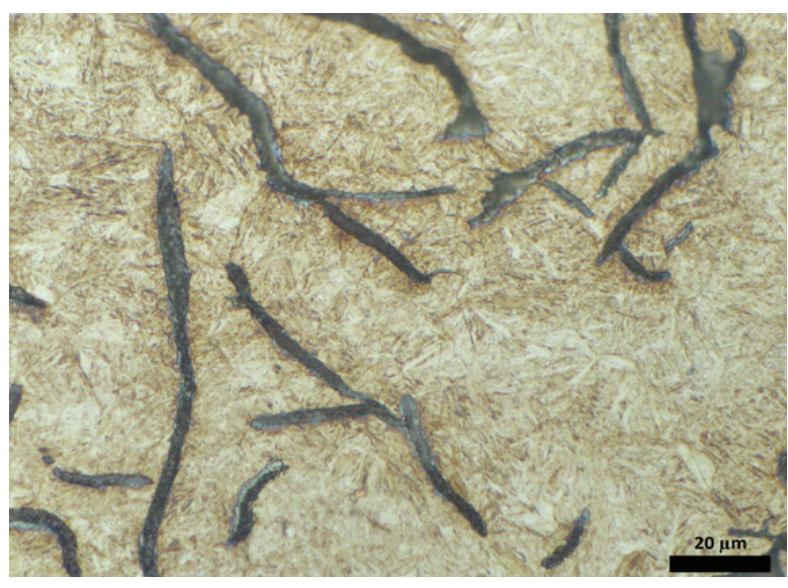

Figura 5. Micrografía de la fundición gris templada y revenida a $200{ }^{\circ} \mathrm{C}$ a $1000 \mathrm{X}$.

fundición gris que fue objeto de estudio, presentaba una matriz de perlita fina con esteadita y grafito laminar tipo $\mathrm{C}$, con rosetas de grafito y grafito eutéctico tipo B, de tamaño, forma y distribución apropiada.

- El estudio de la curva de templabilidad facilita la predicción del comportamiento de la fundición gris al someterse a las condiciones críticas del proceso y está relacionado con la dureza máxima alcanzada durante el enfriamiento, la cual es función del contenido de carbono disuelto en la austenita y los elementos de aleación que retardan la transformación perlítica o ferrítica.

- El tratamiento térmico de temple realizado a la fundición gris CL, permitió una formación de austenita homogénea, que con el enfriamiento posterior originó la microestructura mixta de martensita revenida y austenita retenida con la presencia de grafito libre que se mantiene estable, sin la presencia de señales de distorsión o agrietamiento del material.

\section{AGRADECIMIENTOS}

Los autores expresan su agradecimiento al apoyo económico de COLCIENCIAS (Proyecto $\mathrm{N}^{\circ}$ 467859937197) durante la realización de este trabajo.

\section{REFERENCIAS}

Angus, H.T. (1976). Cast Iron: Physical and Engineering Properties. Second Edition, Elsevier Ltd.

ASM Handbook (1991). Heat Treating. Handbook Committee. Vol. 4, ASM International.

ASM Handbook (1992). Castings. Handbook Committee. Vol. 15, ASM International.

ASTM E3-11 (2011). Standard Guide for Preparation of Metallographic Specimens. ASTM International, West Conshohocken, PA. http://dx.doi.org/10.1520/E0003-11.

ASTM A255-10 (2014). Standard Test Methods for Determining Hardenability of Steel. ASTM International, West Conshohocken, PA. http://dx.doi.org/10.1520/A0255-10R14. 
ASTM E7-15 (2015). Standard Terminology Relating to Metallography. ASTM International, West Conshohocken, PA.

ASTM E407-07 (2015). Standard Practice for Microetching Metals and Alloys, ASTM International, West Conshohocken, PA. http://dx.doi.org/10.1520/E0407-07R15E01.

ASTM A247-16a (2016). Standard Test Method for Evaluating the Microstructure of Graphite in Iron Castings. ASTM International, West Conshohocken, PA. http://dx.doi. org/10.1520/A0247-16A

Balachandran, G., Vadiraj, A., Kamaraj, M., Kazuya, E. (2011). Mechanical and wear behavior of alloyed gray cast iron in the quenched and tempered and austempered conditions. Mater. Design 32 (7), 4042-4049. http://dx.doi. org/10.1016/j.matdes.2011.03.054.

Bates, C.E., Totten, G.H. (1992). Quench Severity Effects on the As-Quenched Hardness of Selected Alloy Steels. Heat Treat. Met. 2, 45-48.

Brooks, C.R. (1979). Heat treatment of ferrous alloys. Editorial McGraw- Hill Book Company, USA

Clarke, K.D., Van Tyne, C.J. (2005). Effect of Prior Microstructure and Heating Rate on Austenite Formation Kinetics in Three Steels for Induction Hardened Components. Report: Department of Metallurgical and Materials Engineering, Colorado School of Mines, USA. https://www.forging.org/ uploaded/content/media/Microstructure_heating.pdf.

Cunningham, J.L., Medlin, D.J., Krauss, G. (1999). Effects of induction hardening and prior cold work on a microalloyed medium carbon steel. J. Mater. Eng. Perform. 8 (4), 401-408. http://dx.doi.org/10.1361/105994999770346684.

Davis, J.R. (1996). Cast Irons ASM Specialty Handbook. ASM International, Handbook Committee.

Dimitry, V.B., Carvalho, M.M.O., de Castro, J.A., Lourenco, T.R.M. (2016). Kinetic Study on Martensite Formation in Steels 1045 and 4340 under Variable Cooling Rates. Mat. Sci. Forum 869, 550-555. http://dx.doi.org/10.4028/www. scientific.net/MSF.869.550.

DPN (2007). Metalmecánica y Siderurgia. Agenda interna para la productividad y competitividad, Departamento Nacional de Planeación, Documento sectorial. https://es.scribd. com/document/214292177/Metalmecanica-y-Siderurgia.

Ferreira, J.C. (2002). A study of cast chilled iron processing technology and wear evaluation of hardened gray iron for automotive application. J. Mater. Process. Tech. 121 (1), 94-101. http://dx.doi.org/10.1016/S0924-0136(01)01208-0.

Gliner, R.E., Vybornov, V.V. (2014). Use of the Standard End Quenching Test for Predicting Heat-Hardening of Cast Iron. Metal Sci. Heat Treat. 56 (7), 424 427. http://dx.doi. org/10.1007/s11041-014-9775-0.
Grossman, M.A. (1942). Hardenability Calculated from Chemical Composition. AIME Transactions 150, 227-259.

Lamont, J.L. (1943). How to estimate hardening depth in bars. Iron Age 152, 64-70.

Lasheras, J.M. (1978). Tecnología del acero. Editorial Cedel, Barcelona, España.

Malinochka, Y.N. (1963). Austenizing gray cast iron. Met. Sci. Heat Treat. 5 (11), 640-646. http://dx.doi.org/10.1007/ $\mathrm{BF} 00660555$.

Maroni, P.J. (1976). Templabilidad: Un método para seleccionar aceros. Editorial librería Mitre, Buenos Aires, Argentina.

Michalski, J., Woś, P. (2011). The effect of cylinder liner surface topography on abrasive wear of piston-cylinder assembly in combustion engine. Wear 271 (3-4), 582-589. http:// dx.doi.org/10.1016/j.wear.2010.05.006.

Piyapong, M. (2007). Solidification modeling of iron castings using solid cast. ProQuest, West Virginia University, USA.

Rejowski, E.D., Soares, E., Roth, I., Rudolph, S. (2012). Cylinder Liner in Ductile Cast Iron for High Loaded Combustion Diesel Engines. J. Eng. Gas Turb. Power. 134 (7), 2807-2815. http://dx.doi.org/10.1115/1.4006071.

Ruiz, J., López, V., Fernández, B.J. (1996). Effect of surface laser treatment on the microstructure and wear behavior of grey iron. Mater. Design 17 (5-6), 267-273. http://dx.doi. org/10.1016/S0261-3069(97)00020-4.

Smolijan, B., Tomasic, N., llikic, D., Felde, I., Reti, T. (2006). Application of Jominy test in 3D simulation of quenching. J. Achiev. Mater. Manuf. Eng. 17 (1-2), 281-284. http://citeseerx.ist.psu.edu/viewdoc/download?doi=10.1.1.548.9283 \&rep $=$ rep1\&type $=$ pdf

Telejko, I., Adrian, H., Skalny, K., Pakiet, M., Staśko, R. (2009). The investigation of hardenability of low alloy structural cast steel. J. Achiev. Mater. Manuf. Eng. 37 (2), 480-485. http://jamme.acmsse.h2.pl/papers_vol37_2/37236.pdf.

Totten, G.E., Bates, C.E., Clinton, N.A. (1993). Handbook of Quenchants and Quenching Technology. ASM International, Materials Park, Ohio, USA.

Vadiraj, A., Balachandran, G., Kamaraj, M., Kazuya, E. (2011). Mechanical and wear behavior of quenched and tempered alloyed hypereutectic gray cast iron. Mater. Design 32 (4), 2438-2443. http://dx.doi.org/10.1016/j. matdes.2010.11.052.

Valencia, A. (2009). Tecnología del tratamiento térmico de los metales. Editorial Universidad de Antioquia, Colombia.

Yamazaki, T., Shibuya, T., Jin, C.J., Kikuta, T., Nakatani, N. (2006). Lining of hydraulic cylinder made of cast iron with copper alloy. J. Mater. Process. Technol. 172 (1), 30-34. http://dx.doi.org/10.1016/j.jmatprotec.2005.08.013. 